\title{
Two-Photon Lif On The Hit-Si3 Experiment: Absolute Density And Temperature Measurements Of Deuterium Neutrals
}

\author{
Drew Elliott \\ Derek Sutherland \\ Umair Siddiqui \\ Earl Scime \\ Chris Everson
}

See next page for additional authors

Follow this and additional works at: https://researchrepository.wvu.edu/faculty_publications

\section{Digital Commons Citation}

Elliott, Drew; Sutherland, Derek; Siddiqui, Umair; Scime, Earl; Everson, Chris; Morgan, Kyle; Hossack, Aaron; Nelson, Brian; and Jarboe, Tom, "Two-Photon Lif On The Hit-Si3 Experiment: Absolute Density And Temperature Measurements Of Deuterium Neutrals" (2016). Faculty \& Staff Scholarship. 715. https://researchrepository.wvu.edu/faculty_publications/715

This Article is brought to you for free and open access by The Research Repository @ WVU. It has been accepted for inclusion in Faculty \& Staff Scholarship by an authorized administrator of The Research Repository @ WVU. For more information, please contact researchrepository@mail.wvu.edu, emily.fidelman@mail.wvu.edu. 


\section{Authors}

Drew Elliott, Derek Sutherland, Umair Siddiqui, Earl Scime, Chris Everson, Kyle Morgan, Aaron Hossack, Brian Nelson, and Tom Jarboe 


\title{
Two-photon LIF on the HIT-SI3 experiment: Absolute density and temperature measurements of deuterium neutrals
}

\author{
Drew Elliott, ${ }^{1, a)}$ Derek Sutherland, ${ }^{2}$ Umair Siddiqui, ${ }^{1}$ Earl Scime, ${ }^{1}$ Chris Everson, ${ }^{2}$ \\ Kyle Morgan, ${ }^{2}$ Aaron Hossack, ${ }^{2}$ Brian Nelson, ${ }^{2}$ and Tom Jarboe ${ }^{2}$ \\ ${ }^{1}$ Department of Physics and Astronomy, West Virginia University, Morgantown, West Virginia 26056, USA \\ ${ }^{2}$ Department of Aerospace Engineering, University of Washington, Seattle, Washington 98105, USA
}

(Presented 8 June 2016; received 31 May 2016; accepted 20 June 2016; published online 20 July 2016)

\begin{abstract}
Two-photon laser-induced fluorescence measurements were performed on the helicity injected torus (HIT-SI3) device to determine the density and temperature of the background neutral deuterium population. Measurements were taken in $2 \mathrm{~ms}$ long pulsed plasmas after the inductive helicity injectors were turned off. Attempts to measure neutrals during the main phase of the plasma were unsuccessful, likely due to the density of neutrals being below the detection threshold of the diagnostic. An unexpectedly low density of atomic deuterium was measured in the afterglow; roughly 100 times lower than the theoretical prediction of $10^{17} \mathrm{~m}^{-3}$. The neutral temperatures measured were on the order of $1 \mathrm{eV}$. Temporally and spatially resolved neutral density and temperature data are presented. Published by AIP Publishing. [http://dx.doi.org/10.1063/1.4955494]
\end{abstract}

\section{INTRODUCTION}

Neutral particle dynamics play an important role in the fusion plasma edge and must be considered to create accurate predictive models of such systems. Neutrals have a large impact on fueling, energy, and momentum transport, and thus particle confinement. Changes in the neutral density in the plasma edge are known to affect critical bulk plasma properties such as the $\mathrm{L}$ to $\mathrm{H}$ transition in tokamaks.

Common ways to measure neutral density in warm (1-20 eV), low $\mathrm{Z}$ plasmas are through Balmer-alpha optical emission spectroscopy (OES) or through fast ion gauges located outside of the plasma. Lying outside of the plasma, fast ion gauges provide no information about the radial profile of neutrals and they also convolve temperature and density information. Determination of the density of neutrals from OES requires a collisional radiative model (CRM) and thus accurate knowledge of the electron energy distribution function throughout the region of interest. Such measurements are notoriously difficult in the edge of fusion plasmas and compound errors through convolution.

Non-resonant laser-induced fluorescence (LIF) solves many of these issues. The intensity of fluorescence is directly proportional to the density of the absorbers (source). By measuring the emission intensity versus pump laser frequency, the velocity distribution (VD) of absorbers is measured. LIF is spatially localized to the region where the fluorescence is collected, often less than $1 \mathrm{~cm}^{3}$. In LIF measurements using pulsed lasers, the temporal resolution is often limited by the repetition rate of the laser and its frequency scan rate. If

Note: Contributed paper, published as part of the Proceedings of the $21 \mathrm{st}$ Topical Conference on High-Temperature Plasma Diagnostics, Madison, Wisconsin, USA, June 2016.

a) Author to whom correspondence should be addressed. Electronic mail: dbelliott@mix.wvu.edu. the plasma is also pulsed, time resolution is recovered by measuring successive plasma pulses.

With LIF, all electronic states, including the ground state which is not accessible via OES, are potentially accessible. For atomic hydrogen and its isotopes, when the local plasma temperature is below $100 \mathrm{eV}$, the ground state represents a majority of the total population. Thus, an LIF measurement of the ground state yields information about the entire neutral population without the need for any modeling. Exciting from the $n=1$ to $n=3$ state with a single photon requires photons well within the vacuum ultraviolet (VUV) range. Therefore twophoton absorption LIF (TALIF) is preferable. For diagnosis of atomic hydrogen and its isotopes, the TALIF pump wavelength is $205 \mathrm{~nm}$ and therefore accessible through vacuum windows. Absolute hydrogenic density measurements are obtained from TALIF after a calibration measurement in a gas of a known density if the relative absorption cross section of the TALIF transition in the calibration species to that in hydrogen is known. In this work, krypton gas is used for the calibration. Figure 1 shows the TALIF energy level schemes used in this work.

Here we present the first spatially and temporally resolved measurements of neutral deuterium velocity distribution (NVDs) in the HIT-SI3 spheromak. These are the first TALIF measurements of neutral density in a fusion class experiment in the USA. The measurements reveal neutral densities much different than those predicted. These measurements underscore the need for accurate neutral diagnostics, to understand particle dynamics in fusion experiments.

\section{EXPERIMENTAL APPARATUS}

The TALIF emission, $S(\lambda)$, from neutrals of velocity space density $n(v)$ is

$$
S(\lambda)=\frac{\Delta \Omega}{4 \pi} n(v) I^{2} \sigma \alpha G,
$$






FIG. 1. The deuterium and krypton TALIF schemes used.

proportional to the square of the laser intensity, $I . \Delta \Omega$ is the solid angle over which the emitted light is collected, $\sigma$ is the absorption cross section from the initial state to the excited state, and $\alpha$, which has a value between 0 and 1 , accounts for the branching ratio from the excited state to the final state of the fluorescence, the transmission efficiency of any optical filters, and the quantum efficiency of the photodetector. The overall gain of the photomultiplier tube (PMT) is $G$.

The output of the PMT is integrated over a short interval centered on the laser pulse. To subtract the background signal, the PMT current before and after the laser pulse is sampled for a time interval set by the observed signal peak. The choice of integration window is constrained by the PMT rise time and fall time, and fundamentally by the laser pulse length (10 ns), and the excited state lifetime $\left(16 \mathrm{~ns}^{1}\right)$,

$$
\begin{aligned}
S(\lambda)= & \int_{t_{0}}^{t_{0}+\Delta t_{m}} A(t) d t-\frac{\Delta t_{m}}{2 \Delta t_{B G}} \int_{t_{0}-\Delta t_{B G}}^{t_{0}} A(t) d t \\
& -\frac{\Delta t_{m}}{2 \Delta t_{B G}} \int_{t_{0}+\Delta t_{m}}^{t_{0}+\Delta t_{m}+\Delta t_{B G}} A(t) d t .
\end{aligned}
$$

$A(t)$ is the PMT current, $t_{0}$ is the start of the laser pulse, and $\Delta t_{m}=50 \mathrm{~ns}$ is the integration time window used in this analysis. Equal background sampling measurement times are used, $\Delta t_{B G}=\Delta t_{m}$, for simplicity. The most significant plasma density oscillations of the HIT-SI3 system occur at the frequency of the helicity injectors, $55.25 \mathrm{kHz}$, and the fastest time scales of the system are on the order of the Alfvén transit time across the minor radius of the device, approximately $1.5 \mu \mathrm{s}$. Because sampling time is much smaller than these characteristic oscillation periods, the background contributions are treated as constant throughout the sampling interval.

Because TALIF is a two-photon process without an intermediate, single photon allowed transition, the absorption cross sections, $\sigma$, for TALIF transitions are much smaller than for LIF transitions. Therefore, TALIF requires high intensity, short pulse lasers. The laser used in these studies was a frequency-tripled, pulsed dye laser pumped with an frequencydouble Nd:YAG laser operating at $20 \mathrm{~Hz}$. The YAG laser generated up to $750 \mathrm{~mJ}$ of $532 \mathrm{~nm}$ light in a $10 \mathrm{~ns}$ pulse. The $532 \mathrm{~nm}$ light pumped a Cobra-Stretch ${ }^{\mathrm{TM}}$ dye laser optimized for producing narrow line width $612-630 \mathrm{~nm}$ light of up to $120 \mathrm{~mJ}$ per pulse. The dye laser pulses were $12 \mathrm{~ns}$ long and were then tripled via a second harmonic generation followed by a sum harmonic generation crystal to produce up to $4 \mathrm{~mJ}$ of 204-210 $\mathrm{nm}$ light in a 6-8 $\mathrm{ns}$ pulse with a $5 \mathrm{~mm}$ beam diameter. All of these measurements reported were obtained with less than $2 \mathrm{~mJ}$ of UV energy.
The relative TALIF absorption cross sections between the krypton and the deuterium schemes, $\left(\sigma_{D e u} / \sigma_{K r}\right)$, is known to be $0.62 .^{2-4}$ Therefore, by comparing separate TALIF measurements performed on krypton gas and deuterium plasmas with known fill pressures of krypton and using the same optical system, absolute measurements of the integrated, over velocity, deuterium ground state density are obtained,

$$
n_{D e u}=\int n_{D e u}(v) d v=n_{K r}\left(\frac{S_{n-D e u}}{S_{n-K r}}\right)\left(\frac{\sigma_{K r}}{\sigma_{D e u}}\right) .
$$

Because the optical paths were the same for the krypton and deuterium measurements, the solid angle term disappears from Equation (1). All of the experiments were performed on the HIT-SI3 spheromak operating in deuterium. ${ }^{5}$ Contrary to tokamak or stellarator configurations, spheromak magnetic fields are fully generated by internal plasma currents. HIT-SI3's currents were maintained by three inductive, nonaxisymmetric, helicity injectors which impart a characteristic oscillation on the plasma with a period of $18 \mu$ s. Flows within the plasma vary by $100 \%-200 \%$ on this time scale. ${ }^{6}$ The electron temperatures were estimated to be between 5 and $20 \mathrm{eV}$ and measured electron number densities ${ }^{7}$ were on the order of $10^{18}-10^{19} \mathrm{~m}^{-3}$. All the measurements were performed when the helicity injectors were turned off and the spheromak was resistively decaying.

The TALIF measurements were obtained with a confocal collection scheme (Figure 2) where both the emitted and injected photons pass through the same focusing/collecting lens. This confocal system used a mirror with a $1 \mathrm{~cm}$ diameter hole cut through the center at a $45^{\circ}$ angle. The mirror allowed the laser light, with its beam diameter of $5 \mathrm{~mm}$, to pass while still reflecting a majority of the collected fluorescent emission towards the PMT. The measurement location in the chamber was changed by moving only the final focusing lens. The only optical component changed between the $\mathrm{Kr}$ calibration measurements and the D measurements was the optical filter.

Measurement was attempted with and without a spatial filter in the collection optics. The spatial filter, an adjustable iris, was intended to reduce the amount of background light entering the PMT. After optimization of the optics during calibration and with the use of the spatial filter, no D signal was measured in the plasma, regardless of other system parameters.

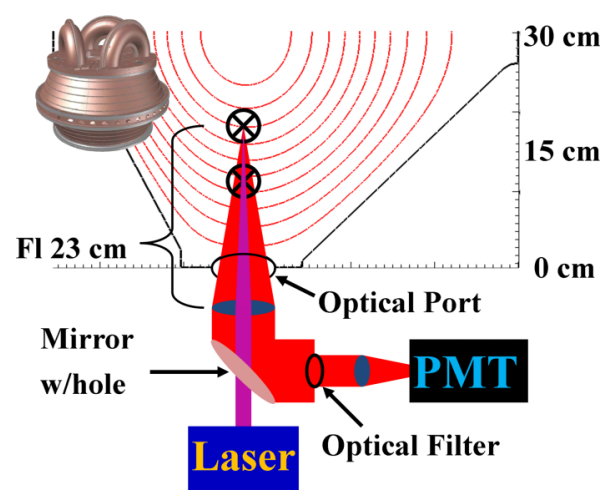

FIG. 2. TALIF in HIT-SI3 configuration showing the external boundary of the vacuum chamber, closed flux surfaces, and the two measurement locations (X's). Inset is a CAD rendering of HIT-SI3. 
A likely explanation for the lack of D signal when using the spatial filter is the differences in focal properties of the optics at the different fluorescence wavelengths.

Because of the confocal injection/collection and TALIF's quadratic dependence on laser intensity, these measurements were well localized. The resolution along the laser path is less than $1 \mathrm{~cm}$, based on beam waist and the quadratic dependence of fluorescence on incident light intensity. Based on beam waist measurements, the off-axis resolution is sub-millimeter.

\section{NEUTRAL DENSITY IN HIT-SI3}

The Kr calibration measurements were obtained with a pressure of 0.2-0.4 mTorr in the HIT-SI3 chamber. The widths of $\mathrm{Kr}$ spectra were anomalously wide due to isotopic broadening, as reported by Magee, so the widths of those velocity distributions do not correspond to the temperature of the neutral gas. ${ }^{12}$ For each measurement location, a new $\mathrm{Kr}$ calibration was performed. The signal-to-noise ratio (SNR) of the $\mathrm{Kr}$ measurements were excellent. Therefore, the dominant source of error in the absolute D measurements was the SNR of the D TALIF measurements.

At a given spatial location and a fixed time index in the decay phase of HIT-SI3 discharges, six separate TALIF measurements were obtained for at least five wavelengths. A sample TALIF signal versus time measurement for a single wavelength in a single HIT-SI3 discharge is shown in Figure 3. The PMT is gated on for only $1 \mu \mathrm{s}$ for each measurement and the laser is triggered at the center of the gate interval to eliminate switching noise and PMT saturation. The PMT gate interval was moved to different times in the HIT-SI3 discharge to explore the time evolution of the neutral D density in the discharge. In Figures 3(a) and 3(c), the laser was tuned to a wavelength off the absorption line whereas in Figures 3(b) and 3(d), the laser was tuned to the peak of the D absorption line. The entirety of the TALIF signal pulse falls within the $50 \mathrm{~ns}$ integration window.

A typical deuterium TALIF signal versus laser wavelength plot is shown in Figure 4. All of the deuterium TALIF (a)

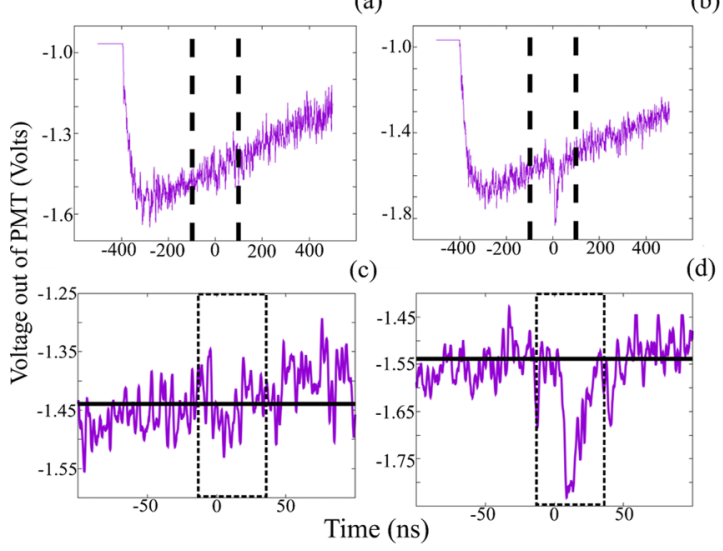

FIG. 3. The PMT signal versus time for the PMT gate "on" interval for (a) a laser pulse tuned to a wavelength outside of the absorption line width and (b) with a laser pulse on resonance. The integration window intervals are magnified in (c) and (d). The thick line in (c) and (d) is the averaged background.

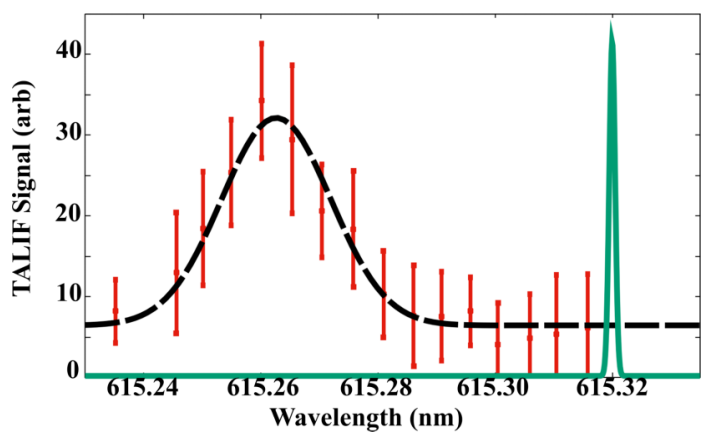

FIG. 4. Normalized deuterium TALIF signal versus wavelength. The curve to the right of the figure is the laser line width on the same scale as the data.

NVD measurements have a signal-to-noise ratio (SNR), peak signal to standard deviation of signal, of approximately $4: 1$. A Maxwellian distribution was fit to the NVD and the area under the curve (normalized by the laser intensity, the PMT gain, and the quantum efficiency of the various filters and detector) yields the density of the deuterium ground state. The width of the fit, $\Delta \lambda$, gives the temperature through $T$ $=\Delta \lambda^{2} m_{D} c^{2} / 2 \lambda_{0}^{2} \ln (2)$. The measured spectral width of Figure 4 is $\Delta \lambda=9.35 \mathrm{pm}$, much higher than the laser linewidth. Thus, the measured broadening is estimated to be entirely thermal. The resultant neutral densities and temperatures at $11 \mathrm{~cm}$ are shown in Figure 5 for different times in the decay phase of the discharge. The TALIF measurements show no evidence of neutral flows. The $\sim 4 \mathrm{pm}$ spacing in measured wavelengths corresponds to a flow resolution of $\sim 2 \mathrm{~km} / \mathrm{s}$. The laser system was capable of measurements with much finer spectral resolution, but to increase the data collection rate spectral resolution was sacrificed.

Although attempts were made to collect data during the main helicity injection phase, no measurable TALIF signal was observed. The absolute densities measured in the afterglow were approximately $10^{15} \mathrm{~m}^{-3}$ and neutral temperatures ranged from 0.83 to $1.70 \mathrm{eV}$. These measurements suggest that the neutral density during the main phase of the discharge is less than $10^{15} \mathrm{~m}^{-3}$ at the positions investigated. This measured value is very near the predicted sensitivity limit of the TALIF diagnostic predicted by Magee et al. ${ }^{8}$ and spans nearly the entire density range predicted in UEDGE simulations of the

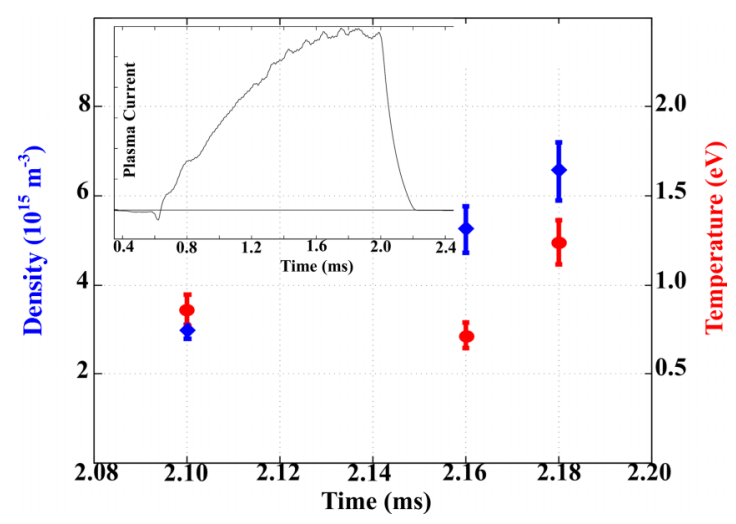

FIG. 5. Absolute deuterium density (diamonds) and temperature (circles) versus time in the decay phase of the discharge at $11 \mathrm{~cm}$. Full discharge current versus time shown in inset image. 


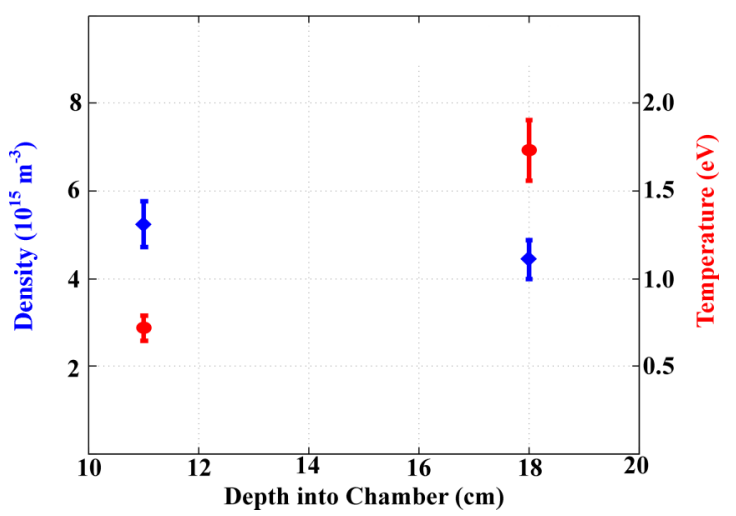

FIG. 6. Absolute deuterium density (diamonds) and temperature (circles) versus position at $t=2.16 \mathrm{~ms}$.

DIII-D plasma edge. The neutral density increases by a factor of 3 monotonically, as expected for the decay phase.

Figure 6 shows the difference in density and temperature at the two spatial locations sampled at $2.16 \mathrm{~ms}$ into the discharge. The density varied only slightly, but the temperature more than doubles moving from $11 \mathrm{~cm}$ to $18 \mathrm{~cm}$ from the chamber wall. While a tokamak edge profile is expected to have strong gradients in the neutral density (increasing exponentially at the near the edge) and such a gradient is not observed here, the positions at which measurements were taken do not represent the edge of the HIT-SI3 device. These measurements were obtained well within the closed flux region of HIT-SI3. Such a region of the device would not be expected to have strong gradients in neutral density.

The errors shown in Figures 5 and 6 are based on the uncertainties in the fits to each measured spectrum. Smaller errors, and therefore smaller uncertainties in the measured densities, are obtainable with an increase in the number of laser pulses per wavelength or with an increase in the number of wavelengths. If the velocity distribution is non-Maxwellian, the error in the temperature may not decrease as a single Maxwellian distribution was assumed in the fits. All of the neutral temperatures recorded in Figures 5 and 6 are on the order of $1 \mathrm{eV}$. This is an order of magnitude smaller than the ion temperatures during the helicity injection phase. ${ }^{7}$

\section{SUMMARY}

These measurements demonstrate that hydrogenic TALIF with a ns pulsed laser is capable of resolving neutral densities as low as $10^{15} \mathrm{~m}^{-3}$ with high spatial and temporal resolution in a short pulse fusion-class experiment. Neutral temperatures ranging from a fraction of an $\mathrm{eV}$ to almost $2 \mathrm{eV}$ were observed. A SNR of 4:1 was achieved with six laser shots per wavelength and an average laser pulse energy of $1 \mathrm{~mJ}$. Without improving any parameters of the diagnostic, neutral density and temperature measurements for every $1.5 \mathrm{~s}$ are feasible using 5 wavelength points and averaging over 6 laser pulses per wavelength. Thus, in a long-pulse tokamak or a steady-state system such as a stellarator, this TALIF diagnostic is capable of providing edge fueling or even isotopic ratio measurements. Improved measurement rates are easily achievable with a higher pulse repetition rate pump laser ${ }^{9}$ or with use of a higher-energy laser pulse.

In addition to demonstrating the feasibility of the TALIF confocal diagnostic on short pulse fusion devices, these initial measurements suggest that simple models used to predict the neutral density in HIT-SI3 are incomplete. Calculations based on plasma resistivity and temperature predict neutral densities $^{7}$ in the range of $10^{17}-10^{19} \mathrm{~m}^{-3}$. While the neutral density measurements presented here have uncertainties as large as $25 \%$, the predicted values were 100 times larger. The lack of TALIF signal earlier in the HIT-SI pulse suggests that much lower neutral densities exist during the main phase.

The impact of these changes in energy and momentum loss processes on our understanding of HIT-SI3 plasmas will be studied in detail with simulations using an extended-MHD code called PSI-TET. ${ }^{10}$ These measurements will be instrumental in validating the dynamic neutral model ${ }^{11}$ being implemented in PSI-TET. Additionally, the measured neutral density provides an approximate lower bound on the electron temperatures expected during the plasma main phase. The estimated value for the electron temperature, assuming a neutral density at or below the TALIF sensitivity threshold of $\sim 2$ $\times 10^{14} \mathrm{~m}^{-3}$, is $T_{e} \geq 12 \mathrm{eV}$. These initial measurements demonstrate that absolutely calibrated neutral diagnostics, such as TALIF, are necessary for complex fusion experiments that seek to develop understanding of underlying neutral dynamics.

The issues in obtaining TALIF measurements in D after optimizing the spatial filter with $\mathrm{Kr}$ identified a significant problem with the $\mathrm{Kr}$ calibration scheme. A calibration scheme employing a xenon transition solves this and is described in another manuscript in this issue. ${ }^{12}$

\section{ACKNOWLEDGMENTS}

This work was supported by Department of Energy Award No. DE-SC0004736, Office of Fusion Energy Science Award No. DE-FG02-96ER54361e and NSF Award No. PHYS1360278.

${ }^{1}$ T. Kajiwara, T. Shinkawa, K. Uchino, M. Masuda, K. Muraoka, T. Okada, M. Maeda, S. Sudo, and T. Obiki, Rev. Sci. Instrum. 62, 2345-2349 (1991).

${ }^{2}$ M. G. H. Boogaarts, S. Mazouffre, G. J. Brinkman, H. W. P. van der Heijden, P. Vankan, J. A. M. van der Mullen, D. C. Schram, and H. F. Döbele, Rev. Sci. Instrum. 73, 73-86 (2002).

${ }^{3}$ J. Bokor, R. Freeman, J. White, and R. Starz, Phys. Rev. A 24, 612 (1981).

${ }^{4}$ K. Niemi, V. Schulz-von der Fathen, and H. F. Dobele, J. Phys. D: Appl. Phys. 34, 2330 (2001).

${ }^{5}$ T. R. Jarboe, C. J. Hansen, A. C. Hossack, G. J. Marklin, K. D. Morgan, B. A. Nelson, D. A. Sutherland, and B. S. Victor, Fusion Sci. Technol. 66, 369-384 (2014).

${ }^{6}$ D. A. Sutherland, T. R. Jarboe, K. D. Morgan, M. Plaff, E. S. Lavine, Y. Kamikawa, M. Hughes, P. Andrist, G. Marklin, and B. A. Nelson, Fusion Eng. Des. 89, 412 (2014).

${ }^{7}$ A. Hossack, Ph.D. thesis, University of Washington, 2015.

${ }^{8}$ R. M. Magee, M. E. Galante, D. McCarren, E. E. Scime, R. L. Boivin, N. H. Brooks, R. J. Groebner, D. N. Hill, and G. D. Porter, Rev. Sci. Instrum. 83, 10D701 (2012).

${ }^{9}$ D. Den Hartog, N. Jiang, and W. R. Lempert, Rev. Sci. Instrum. 79, 10 E736 (2008).

${ }^{10}$ C. J. Hansen, Ph.D. thesis, University of Washington, 2014.

${ }^{11}$ E. T. Meier and U. Shumlak, Phys. Plasmas 19, 072508 (2012).

${ }^{12}$ D. Elliott, E. Scime, and Z. Short, Rev. Sci. Instrum. 87, 11 E504 (2016). 